\title{
Effect of smectic $A$ temperature width on the soft mode in ferroelectric liquid crystals
}

\author{
A. Choudhary, ${ }^{1,2}$ S. Kaur, ${ }^{2}$ J. Prakash, ${ }^{2}$ K. Sreenivas, ${ }^{1}$ S. S. Bawa, ${ }^{2}$ and A. M. Biradar ${ }^{2, a)}$ \\ ${ }^{1}$ Department of Physics and Astrophysics, University of Delhi, Delhi 110007, India \\ ${ }^{2}$ National Physical Laboratory, Dr. K. S. Krishnan Road, New Delhi 110012, India
}

(Received 22 February 2008; accepted 1 May 2008; published online 7 August 2008)

\begin{abstract}
The behavior of soft mode range with respect to the temperature width of smectic $A(\mathrm{Sm} A)$ phase has been studied in four different ferroelectric liquid crystal (FLC) materials in the frequency range $10 \mathrm{~Hz}-10 \mathrm{MHz}$. The studies have been carried out in a planarly well aligned cells at different temperatures and different bias fields in $\mathrm{Sm} C^{*}$ and $\mathrm{Sm} A$ phases. Dielectric studies of these FLCs near $\mathrm{Sm} C^{*}$-Sm $A$ phase transition show that the temperature range of soft mode relaxation frequency phenomenon varies with the temperature width of $\mathrm{Sm} A$ phase. The dependence of tilt angle on temperature shows the nature of the order of transition at $\operatorname{Sm} C^{*}$-Sm $A$ phase. The coupling between order parameters of $\mathrm{Sm} C^{*}$ and $\mathrm{Sm} A$ phase influences the soft mode and phase transition in Sm $C^{*}$ and Sm A phases. (C) 2008 American Institute of Physics. [DOI: 10.1063/1.2952056]
\end{abstract}

\section{INTRODUCTION}

After the discovery of ferroelectric liquid crystals (FLCs) ${ }^{1}$ the complex structure and unique properties of these materials, especially near transition temperature $\left(T_{c}\right)$ of smectic $C^{*}$-smectic $A\left(\mathrm{Sm} C^{*}\right.$-Sm $\left.A\right)$ phase, have attracted the attention of material researchers. Theoretical as well as experimental studies had been carried out by dielectric spectroscopy in both $\mathrm{Sm} C^{*}$ and $\mathrm{Sm} A$ phases revealing the Goldstone mode in $\mathrm{Sm} C^{*}$ phase due to the phason fluctuations and soft mode in Sm $A$ phase due to tilt fluctuations. ${ }^{2,3}$ The existence of soft mode in $\mathrm{Sm} C^{*}$ phase has been observed by applying strong enough bias voltage to restrict the phason fluctuations or helix unwinding near $T_{c}$.

The observed excess heat capacity in the vicinity of Sm $C^{*}$-Sm $A$ phase transition is well described by adding a sixth order term in Landau's expression of free-energy density, which suggests that it might be due to the fluctuations of $\mathrm{Sm}$ $A$ order near $T_{c}$ of the $\mathrm{Sm} C^{*}-\mathrm{Sm} A$ phase. ${ }^{4-6}$ However, the importance of a sixth order term was confirmed in the study of materials near the $\mathrm{Sm} C^{*}-\mathrm{Sm} A$ transition. ${ }^{7}$ The $\mathrm{Sm}$ $C^{*}$-Sm $A$ phase transition can be well described by the extended mean field theory of free energy. ${ }^{8}$ The transition of $\mathrm{Sm} C^{*}$ to $\mathrm{Sm} A$ phase is influenced by factors such as molecular interaction, dipole moment, and width of $\mathrm{Sm} A$ phase. $^{9-11}$ In general, the $\mathrm{Sm} C^{*}-\mathrm{Sm} A$ phase transition is found to be second order. However, several compounds exhibit the existence of first order phase transition. ${ }^{12,13} \mathrm{X}$-ray studies showed the change in order of transition from second to first order by a mere decrease in the temperature range of Sm $A$ phase. ${ }^{11}$ Compounds having high polarization $\left(P_{s}\right)$ were considered to exhibit a first order $\operatorname{Sm} C^{*}$-Sm $A$ phase transition. ${ }^{12,14,15}$ Experimentally, it was shown that the $\mathrm{Sm}$ $C^{*}$-Sm $A$ phase transition was mainly driven by intermolecular forces producing the $\mathrm{Sm} C^{*}$ phase. ${ }^{16-18}$ However, some

\footnotetext{
${ }^{a)}$ Author to whom correspondence should be addressed. Electronic mail: abiradar@mail.nplindia.ernet.in.
}

compounds having a smaller $\mathrm{Sm} A$ phase $^{10}$ or no $\mathrm{Sm} A$ phase $^{19}$ and small $P_{s}$ have also shown a second order $\mathrm{Sm}$ $C^{*}$-Sm $A$ phase transition. ${ }^{20}$

In the present paper, we report the effect of temperature width of the $\mathrm{Sm} A$ phase on the behavior of soft mode temperature range in the vicinity of the $\mathrm{Sm} C^{*}-\mathrm{Sm} A$ phase transition. Here, the soft mode range means the range of temperature in which the soft mode dielectric relaxation process appears near $T_{c}$ of the $\mathrm{Sm} C^{*}$-Sm $A$ phase. Dielectric studies on all the four FLC materials of varying $\operatorname{Sm} A$ phase temperature width confirm that the regime of soft mode in the $\mathrm{Sm} C^{*}$ and $\mathrm{Sm} A$ phases is influenced by the temperature width of the Sm $A$ phase. In case of a large $\operatorname{Sm} A$ phase width, the soft mode appears both above and below $T_{c}$. On the contrary, reducing the $\mathrm{Sm} A$ phase width, the range of soft mode shrinks towards $T_{c}$ of the $\operatorname{Sm} C^{*}$-Sm $A$ phase. In the present case with a narrow $\mathrm{Sm} A$ phase temperature range material, the possibility of existence of soft mode is minimum in the $\mathrm{Sm} C^{*}$ phase and even in the $\mathrm{Sm} A$ phase. All the materials possess nonsequential $P_{s}$ and pitch value at room temperature. In other words, $P_{s}$ and pitch value of these materials do not follow the order of sequence of the Sm $A$ phase temperature width.

\section{EXPERIMENT}

Four FLC materials with the following phase sequence have been studied:

(1) Felix 17/100: cryst $-28{ }^{\circ} \mathrm{C} \mathrm{Sm} C^{*} 73{ }^{\circ} \mathrm{C} \mathrm{Sm} A 77^{\circ} \mathrm{C}$ $\mathrm{N} 84-87^{\circ} \mathrm{C}$ iso,

(2) CS 1016: cryst $-21{ }^{\circ} \mathrm{C} \mathrm{Sm} C^{*} 56{ }^{\circ} \mathrm{C} \mathrm{Sm} A 67{ }^{\circ} \mathrm{C} \mathrm{N}$ $73{ }^{\circ} \mathrm{C}$ iso,

(3) CS 1026: cryst $-7{ }^{\circ} \mathrm{C} \mathrm{Sm} C^{*} 64{ }^{\circ} \mathrm{C} \mathrm{Sm} \mathrm{A} 82{ }^{\circ} \mathrm{C} \mathrm{N}$ $91{ }^{\circ} \mathrm{C}$ iso, and

(4) Felix 20: cryst $-8{ }^{\circ} \mathrm{C} \mathrm{Sm} C^{*} 15-18{ }^{\circ} \mathrm{C} \mathrm{Sm} A 75{ }^{\circ} \mathrm{C} \mathrm{N}$ 92- $103{ }^{\circ} \mathrm{C}$ iso.

Here, iso and cryst stand for isotropic and crystalline 
phases, respectively. The sample cells were fabricated by using highly conducting $(\sim 15 \Omega / \square)$ indium tin oxide (ITO) coated $\lambda / 2$ glass plates. ITO patterns, which acted as electrodes, were prepared by a negative photoresist lithography technique in the form of squares of $4.5 \times 4.5 \mathrm{~mm}^{2}$ on one electrode and $6 \times 6 \mathrm{~mm}^{2}$ on another to avoid the mismatching of conducting portion. Thus, the effective area under study was $4.5 \times 4.5 \mathrm{~mm}^{2}$. Both the glass plates having a desired pattern were spin coated with adhesion promoter (phenyl trichlorosilane in toluene) followed by nylon (6/6) coating, and were rubbed unidirectionally to get homogeneous alignment. ${ }^{21}$ The thickness between the two plates was maintained using Mylar spacers and was kept more than the pitch value of the materials in order to avoid surface stabilized FLC state. Cells were calibrated using air and toluene as standard references. Materials were filled in by means of capillary action in isotropic phase and then cooled gradually to room temperature.

The samples were viewed under a Carl-Zeiss Axioscope-40 polarizing microscope to confirm the homogeneous alignment. Dielectric measurements were carried out in an electrically shielded parallel plate condenser, ${ }^{21}$ using two bridges: Solartron 1260 and HP 4192A impedance analyzers in the frequency range $10 \mathrm{~Hz}-10 \mathrm{MHz}$. The temperature of the sample cell was controlled by maintaining the temperature of water circulating through the sample holder by a Zulabo temperature controller with an accuracy of $\pm 0.01{ }^{\circ} \mathrm{C}$. Tilt-angle measurements were carried out by mounting the cell under study on a rotatable stage of the polarizing microscope and dc bias voltage was applied. Differential scanning calorimetry was carried out from $\mathrm{Sm} C^{*}$ to isotropic phase using Mettler Toledo differential scanning calorimetry (DSC) $822^{\mathrm{e}}$ at a scanning rate of $2{ }^{\circ} \mathrm{C} / \mathrm{min}$.

\section{RESULTS AND DISCUSSIONS}

\section{A. Effect of $\mathrm{Sm} A$ phase temperature width on the nature of $\mathrm{Sm} C^{*}-\mathrm{Sm} A$ phase transition}

The effect of the Sm $A$ phase width on the nature of the Sm $C^{*}$-Sm $A$ phase transition has been studied by investigating the primary order parameter, i.e., tilt-angle behavior in the vicinity of the $\operatorname{Sm~} C^{*}$-Sm $A$ phase transition of these FLC materials. It has already been reported that the order of the $\mathrm{Sm} C^{*}$-Sm $A$ phase transition can be from second order to first order by decreasing the $\operatorname{Sm} A$ temperature range. ${ }^{7,10,11}$ This is evident from Fig. 1, which shows the variation of tilt angle with temperature. Figure 1(a) shows the behavior of optical tilt as a function of temperature of Felix 20, which has a wide $\mathrm{Sm} A$ phase of $57^{\circ} \mathrm{C}$. One can see that the transition is continuous at the $\mathrm{Sm} C^{*}$-Sm $A$ phase $T_{c}$ $\left(15-18{ }^{\circ} \mathrm{C}\right)$. On the contrary, FLC Felix $17 / 100$ with a Sm $A$ phase width of only $4{ }^{\circ} \mathrm{C}$ shows the discontinuity in the tilt angle at $T_{c}$ of the $\mathrm{Sm} C^{*}$-Sm $A$ phase, as shown in Fig. 1(b) The FLC materials CS 1026 and CS 1016 with Sm A phase widths of 18 and $11^{\circ} \mathrm{C}$, respectively, show a continuous phase transition. Figure 1(b) shows the tilt-angle behavior of CS 1016 while the tilt angle behavior of CS 1026 has already been published elsewhere. ${ }^{22}$ From the above observation, it can be inferred that, indeed, discontinuity in the tilt
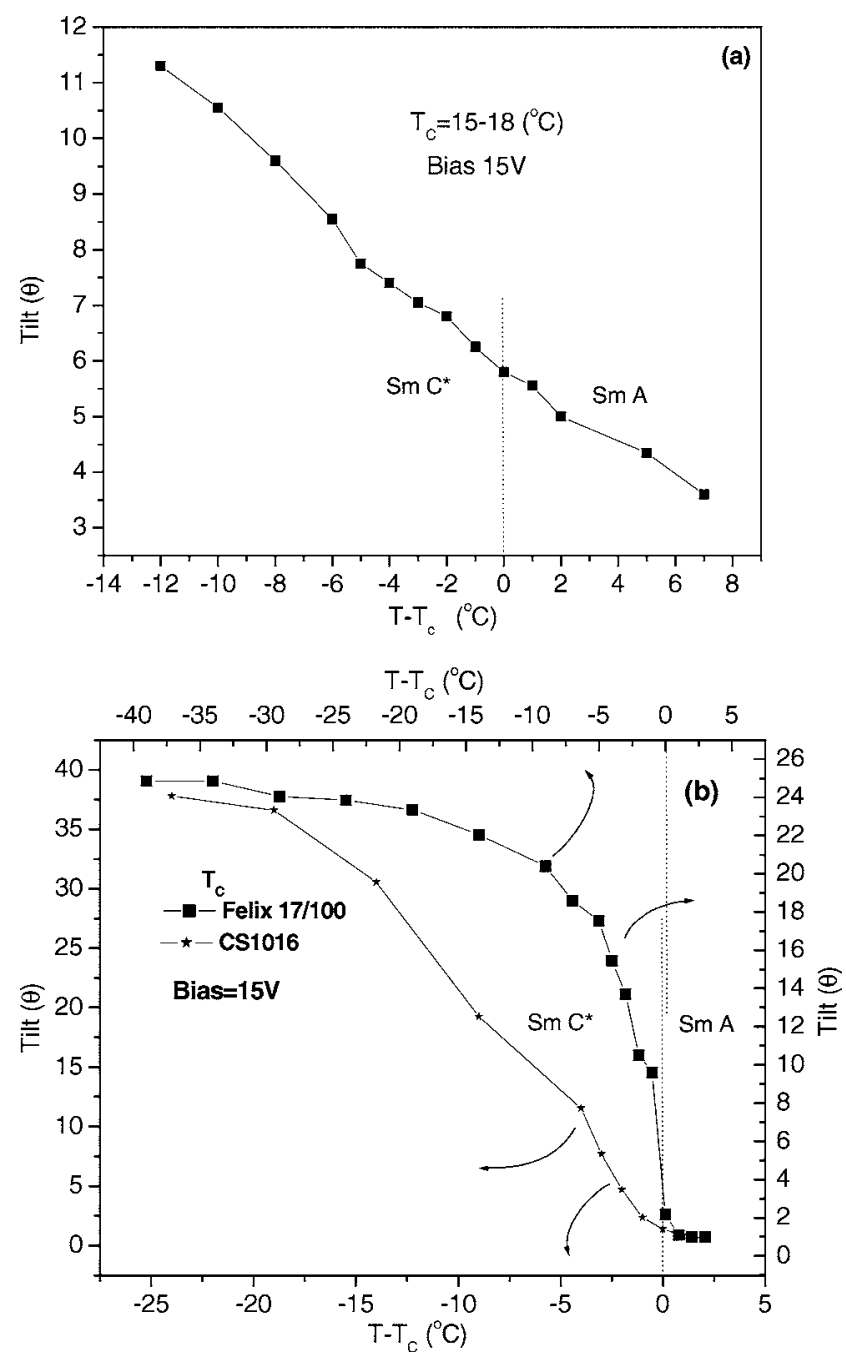

FIG. 1. Behavior of optical tilt $(\theta)$ as a function of bias voltage in case of (a) Felix-20, (b) Felix-17/100, and CS 1016.

angle appears on reducing the $\operatorname{Sm} A$ phase width. It is worth to point out here that all FLCs here possess $C^{*}-A-N$-iso phase transitions.

Further, these materials were studied by DSC to investigate the nature of phase transition and the associated enthalpy change. Figure 2(a) shows the DSC scan obtained for Felix 17/100. The material $(15.2 \mathrm{mg})$ was scanned at a heating rate of $2{ }^{\circ} \mathrm{C} \mathrm{min}^{-1}$. As seen in Fig. 2(a), three peaks have been observed associated with the $\operatorname{Sm} C^{*}$-Sm $A$ phase transition; the second and third peaks are due to the $\operatorname{Sm} A-N$ and $N$-iso phase transitions, respectively. Figure 2(b) shows the DSC heating curves for CS 1026 material $(2.7 \mathrm{mg}$ of material). The two peaks observed in this material are due to the Sm $A-N$ and $N$-iso phase transitions at 82 and $91{ }^{\circ} \mathrm{C}$, respectively. It is difficult to identify the $\operatorname{Sm} C^{*}$-Sm $A$ phase transition peak in this curve [Fig. 2(b)]. In the other two materials (CS 1016 and Felix 20) also, no peak was observed at the Sm $C^{*}$-Sm $A$ phase transition. Consequently, the enthalpy and entropy changes at the $\operatorname{Sm} C^{*}$-Sm $A$ phase transition are much different in Felix 17/100 (having a width of $4{ }^{\circ} \mathrm{C}$ in the Sm A phase) as compared to the other three studied FLC materials where the width of the $\operatorname{Sm} A$ phase temperature is much higher than Felix 17/100. 

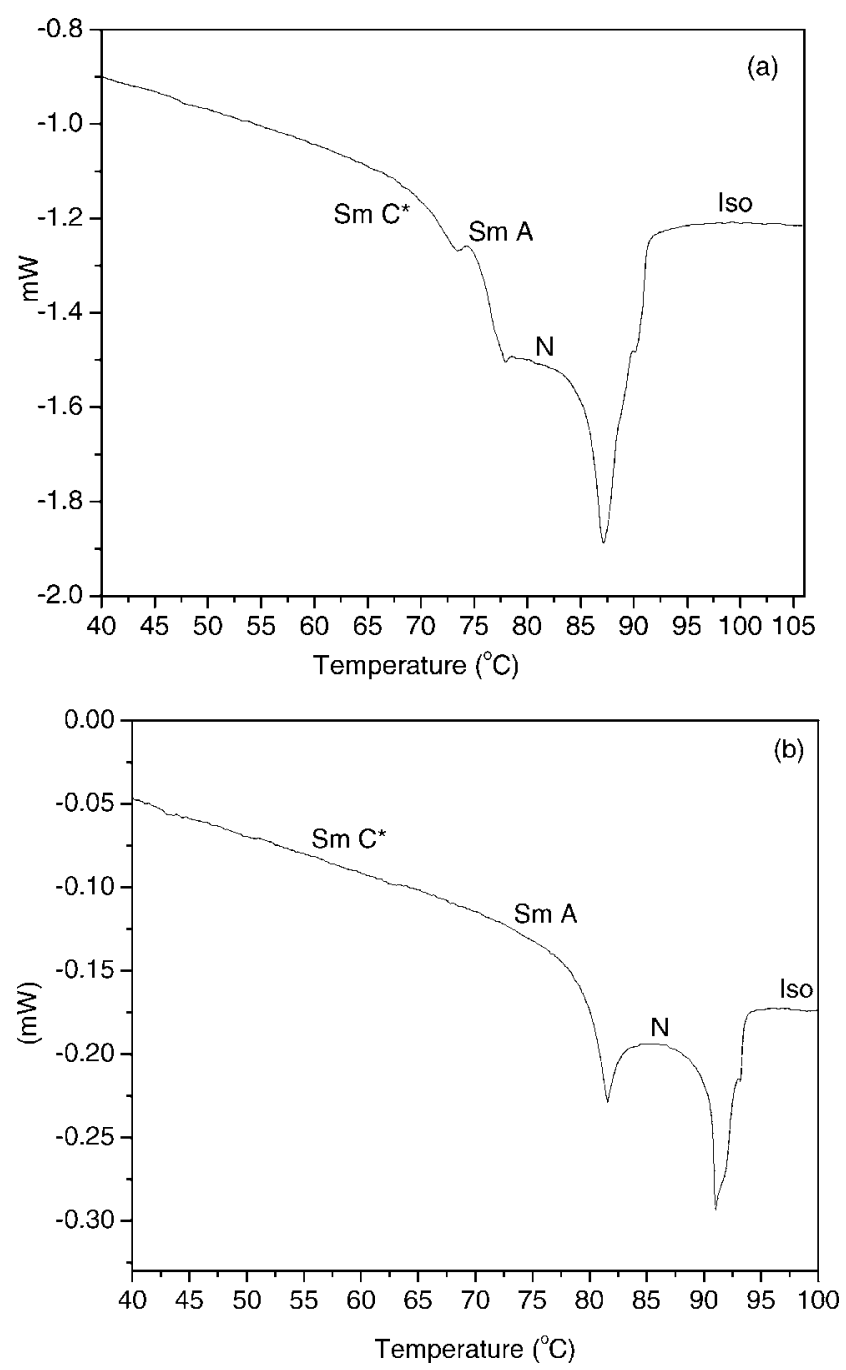

FIG. 2. Differential scanning thermograms obtained on heating for (a) Felix-17/100 and (b) CS 1026 at the rate of $2{ }^{\circ} \mathrm{C} \mathrm{min}^{-1}$.

\section{B. Effect of Sm A phase temperature width on soft mode}

Dielectric studies have been carried out on all the four studied materials having different $\mathrm{Sm} A$ phase temperature ranges and exhibited different observations of soft mode range in $\mathrm{Sm} C^{*}$ and $\mathrm{Sm} A$ phases. Figures 3(a) and 3(b) show the variation of $\varepsilon^{\prime}$ and $\tan \delta$, respectively, at $0 \mathrm{~V}$ as a function of frequency at different temperatures in the CS 1016 material. The frequency dependence of complex dielectric permittivity in the $\mathrm{Sm} C^{*}$ phase can be written as

$$
\varepsilon^{*}(\omega)=\varepsilon_{\infty}+\sum_{i=G, S} \frac{\left(\varepsilon_{0}^{i}-\varepsilon_{\infty}^{i}\right)}{1-\left(i \omega \tau_{i}\right)^{1-\alpha_{i}}},
$$

where $\alpha_{i}$ stands for the distribution parameter, and $G$ and $S$ stands for Goldstone and soft mode, respectively. As shown in Fig. 3(a), the contribution to $\varepsilon^{\prime}$ is dominated by Goldstone mode amplitude at low temperatures in the $\mathrm{Sm} C^{*}$ phase. However, the soft mode can be observed very close to the $\mathrm{Sm} C^{*}$-Sm $A$ phase transition in the $\mathrm{Sm} C^{*}$ phase [Fig. 3(b)]. As observed in the figure, the magnitude of $\varepsilon^{\prime}$ at low frequency decreases with temperature. The high value of $\varepsilon^{\prime}$ at low frequency is due to the Goldstone mode; the contribution of the soft mode to $\varepsilon^{\prime}$ is very low in magnitude as compared to the Goldstone mode.

Figures 3(c) and 3(d) show the permittivity and $\tan \delta$, respectively, as a function of frequency at a bias field of $10 \mathrm{~V}$ at different temperatures. $\varepsilon^{\prime}$ shows an abrupt increase just before the $T_{c}$, which is due to the contribution of soft mode to dielectric permittivity in the $\mathrm{Sm} C^{*}$ phase. Application of high enough $10 \mathrm{~V}$ bias to unwind the helix gives stronger evidence of soft mode in the $\mathrm{Sm} C^{*}$ phase near $T_{c}$. It is known that the amplitude of order parameters is affected by external forces at $T_{c}$ in the form of soft mode, which contributes to the dielectric permittivity. ${ }^{23}$ As seen in Fig. $3(\mathrm{c}), \varepsilon^{\prime}$ increases just $1{ }^{\circ} \mathrm{C}$ below $T_{c}$, attains a maximum value at $T_{c}$, and then starts decreasing up to $2{ }^{\circ} \mathrm{C}$ temperature in the $\mathrm{Sm} A$ phase.

The dielectric absorption at $0 \mathrm{~V}$ bias exhibits the general behavior of Goldstone mode as seen in Fig. 3(b) in the Sm $C^{*}$ and $\mathrm{Sm} A$ phases. However, the behavior of soft mode can be better visualized by applying a bias field of $10 \mathrm{~V}$ and suppressing the Goldstone mode, as observed in Fig. 3(d). The suppressed Goldstone mode is very clearly seen in the $\mathrm{Sm} C^{*}$ phase while the soft mode in both the $\mathrm{Sm} C^{*}$ and $\mathrm{Sm}$ $A$ phases. On the application of a strong $10 \mathrm{~V}$ bias, the dielectric absorption peak near $T_{c}$ in the $\mathrm{Sm} C^{*}$ phase shifts to a high frequency value. Phason fluctuations become weak and get suppressed by the application of strong bias, thereby allowing tilt fluctuations to be observed in the $\mathrm{Sm} C^{*}$ phase near $T_{c}$. Under bias field, in the $\mathrm{Sm} C^{*}$ phase, only the suppressed Goldstone mode is observed whereas beyond $T_{c}$ soft mode relaxation frequency can be seen. The variation of relaxation frequency with temperature at biases of 0 and $10 \mathrm{~V}$ is shown in Fig. 4. At $0 \mathrm{~V}$ bias, constant relaxation frequency shows the temperature independence of Goldstone mode in the $\mathrm{Sm} C^{*}$ phase. At $10 \mathrm{~V}$ bias, an abrupt increase before $T_{c}$ is observed, which is due to tilt fluctuation, i.e., soft mode in the $\mathrm{Sm} C^{*}$ phase. Beyond $T_{c}$, in the $\mathrm{Sm} A$ phase, the increase in relaxation is due to soft mode. The observed temperature widths of soft mode in the $\mathrm{Sm} C^{*}$ and $\mathrm{Sm} A$ phases in CS 1016 material are 0.5 and $1{ }^{\circ} \mathrm{C}$, respectively (Fig. 4).

Another FLC material, Felix 17/100, having a Sm $A$ phase temperature range of $4{ }^{\circ} \mathrm{C}$ was investigated by dielectric spectroscopy. Figure 5(a) shows the temperature and frequency dependence of dielectric permittivity of this material. A decrease in absolute value of $\varepsilon^{\prime}$ with temperature and an abrupt decrease near $T_{c}, 73{ }^{\circ} \mathrm{C}$, have been observed. Phason fluctuations are strong at low frequency and low temperature. On the application of $10 \mathrm{~V}$ dc bias, which is strong enough to unwind the helix, the amplitude of the phason mode is suppressed to a low value due to alignment of dipole moments in the direction of strong dc field or unwinding of helix, as seen in Fig. 5(b). Although there is a slight increment in the absolute value of dielectric permittivity near the $\mathrm{Sm} C^{*}$-Sm $A$ phase transition, which is probably due to the presence of soft mode in a very narrow temperature range, no clear peak of soft mode was observed in the absorption curve as seen in Fig. 5(c). There is no relaxation in the dielectric absorption after $T_{c}$ in the $\operatorname{Sm} A$ phase. As seen in the figure, on the application of strong bias, only suppressed Goldstone mode peak has been observed and there is no 

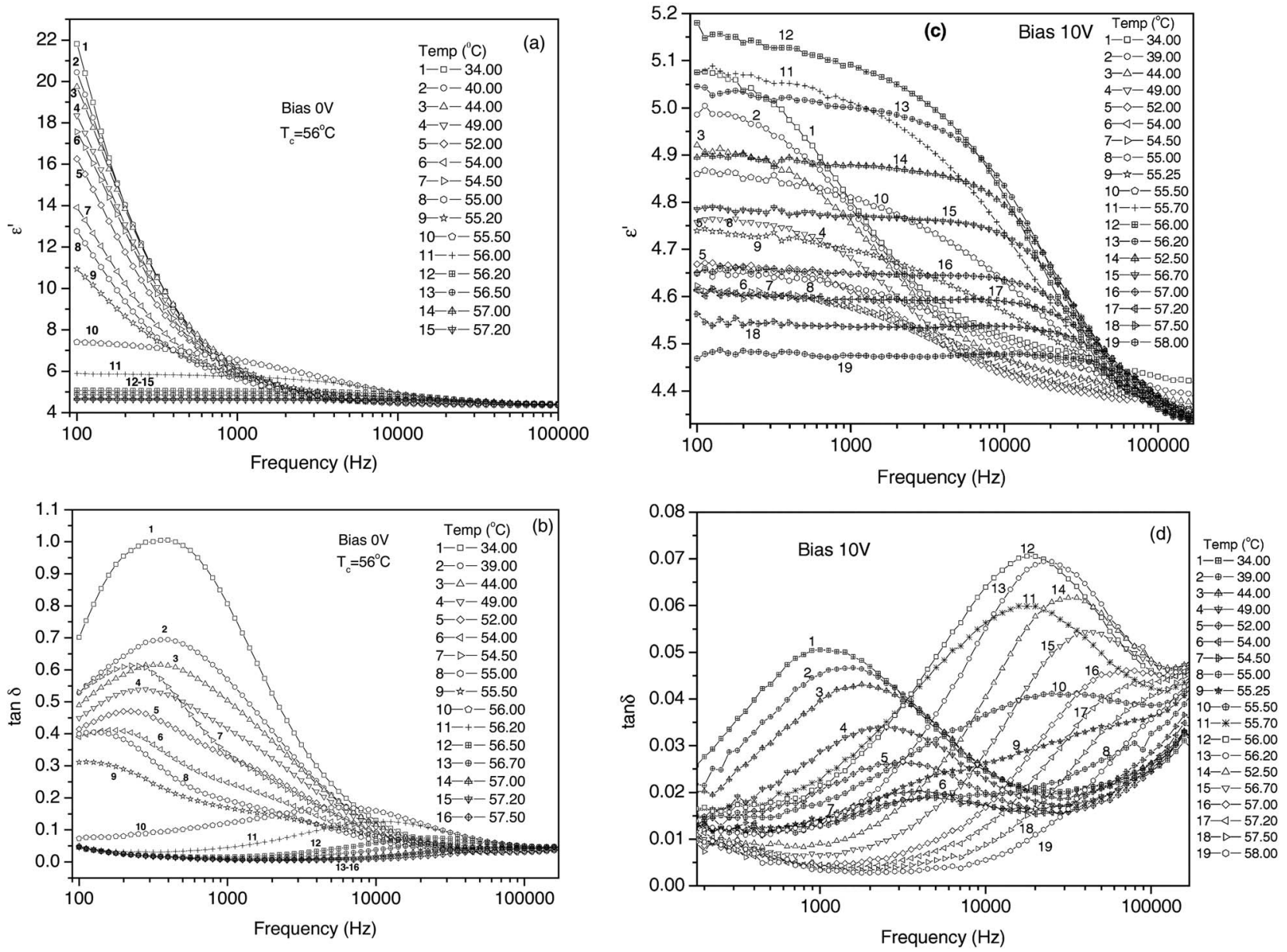

FIG. 3. (a) Dielectric dispersion and (b) dielectric loss factor, as a function of frequency at different temperatures at $0 \mathrm{~V}$ bias. (c) Dielectric dispersion and (d) dielectric loss factor at $10 \mathrm{~V}$ bias in CS 1016 material.

indication of soft mode peak before and after $T_{c}$. The relaxation frequency variation with temperature at $10 \mathrm{~V}$ dc bias is shown in Fig. 5(c). The suppressed Goldstone mode is visualized at $10 \mathrm{~V}$ bias, which disappears at/near $T_{c}$ and the soft mode phenomenon is not observed even in the $\mathrm{Sm} A$ phase. However, at $0 \mathrm{~V}$ bias, a weak decrease in Goldstone mode

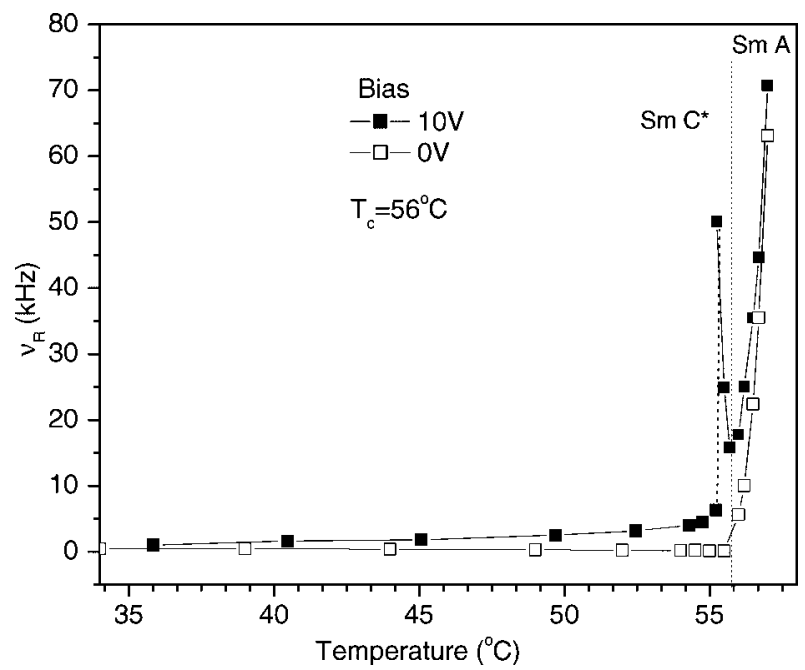

FIG. 4. Temperature dependence of relaxation frequency $\left(v_{R}\right)$ at 0 and $10 \mathrm{~V}$ biases in CS 1016. frequency is observed near $T_{c}$, which is common behavior in FLCs. It is worth mentioning here that at high bias field even suppressed Goldstone mode is not seen near $T_{c}$ as observed from Fig. 5(c), suggesting that phase fluctuations and tilt fluctuations are very weak near $T_{c}$ in this material.

The Sm A phase width of Felix 17/100 is less as compared to CS 1016 . Soft mode in CS 1016 is about $0.5^{\circ} \mathrm{C}$ in $\mathrm{Sm} C^{*}$ and $1{ }^{\circ} \mathrm{C}$ in $\mathrm{Sm} A$ phase whereas in Felix $17 / 100$ with a lower $\mathrm{Sm} A$ phase width, it has not been observed in either of the phases as seen in Fig. 5(c). This gives the indication of influence of the $\operatorname{Sm} A$ phase temperature width on the temperature range of soft mode. In order to confirm this observation of relaxation behavior, two more materials were studied. One having a $\mathrm{Sm} A$ phase temperature width of $57^{\circ} \mathrm{C}$ (Felix 20) while another of $18^{\circ} \mathrm{C}$ (CS 1026).

Felix 20 material $^{21}$ possesses $57^{\circ} \mathrm{C}$ as the width of the $\mathrm{Sm} A$ phase, the largest temperature range among the studied four materials. Figure 6 shows the relaxation frequency with respect to temperature in Felix 20 material. As seen in Fig. 6, the relaxation behavior is temperature dependent in the $\operatorname{Sm} A$ phase beyond $T_{c}\left(15-18{ }^{\circ} \mathrm{C}\right)$ in both the cases of no bias and high dc bias of $25 \mathrm{~V}$. Soft mode phenomenon is observed in a large range of $8{ }^{\circ} \mathrm{C}$ in the $\mathrm{Sm} A$ phase while in the $\mathrm{Sm} C^{*}$ phase it shows a constant relaxation behavior at $25 \mathrm{~V}$ bias. Also, in de Vries electroclinic liquid crystal with a large Sm 

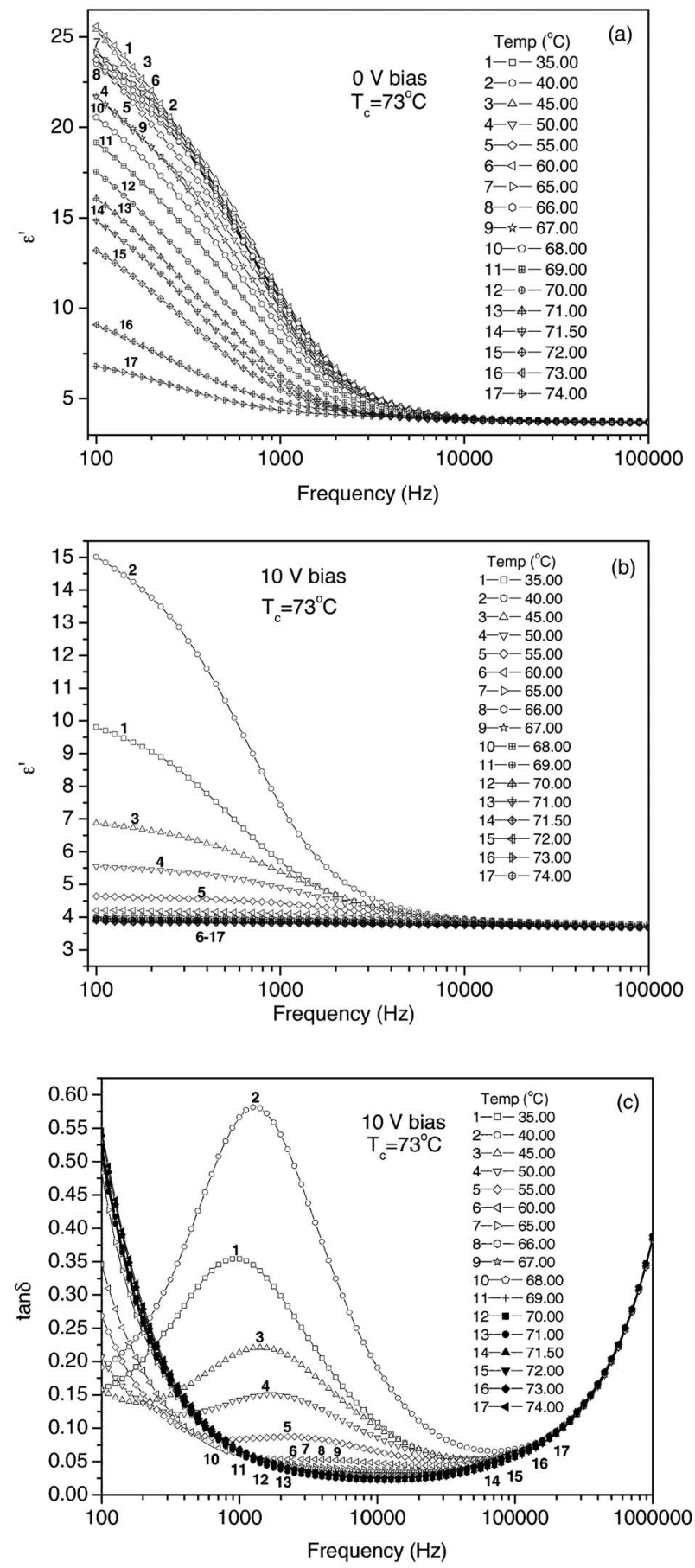

FIG. 5. Dielectric dispersion as a function of frequency at different temperatures at (a) 0 and (b) $10 \mathrm{~V}$ biases. (c) Dielectric loss factor as a function of frequency at different temperatures at $10 \mathrm{~V}$ bias in Felix-17/100.

$A$ phase, the soft mode appearance is observed in a wider temperature range in the Sm $A$ phase. ${ }^{21,22,24}$ Felix 20 is a de Vries type of electroclinic liquid crystal, ${ }^{21}$ as can be observed by the variation in tilt angle with temperature [Fig. 1(a)] and shows a similar behavior as exhibited by other electroclinic liquid crystal BDH 764E. ${ }^{22}$ In BDH 764E, no soft mode has been observed in the $\mathrm{Sm} C^{*}$ phase and the temperature range of soft mode in the $\mathrm{Sm} A$ phase is about $8{ }^{\circ} \mathrm{C}$, which is similar to the behavior shown by Felix 20. Another FLC material CS 1026 having a Sm $A$ temperature width of $18{ }^{\circ} \mathrm{C}$, which is in between the width of CS 1016 and Felix

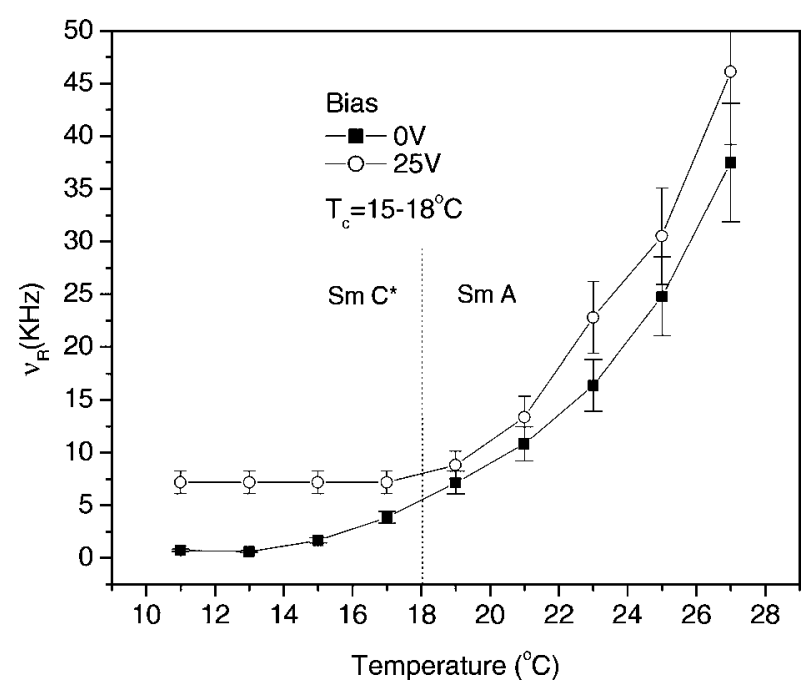

FIG. 6. Temperature dependence of relaxation frequency $\left(v_{R}\right)$ at 0 and $25 \mathrm{~V}$ biases in Felix-20.

20, was also studied to confirm the width of the soft mode near $T_{c}$ temperature. Figure 7 shows the relaxation behavior exhibited by the CS 1026 material. ${ }^{22}$ As seen, the soft mode is observed for $2{ }^{\circ} \mathrm{C}$ in the $\mathrm{Sm} C^{*}$ phase and $3.5^{\circ} \mathrm{C}$ in the $\mathrm{Sm} A$ phase. As discussed, it is clear that the behavior of soft mode near $T_{c}$ is very much dependent on the temperature range of the $\mathrm{Sm} A$ phase, implying that materials having a wide temperature width of the $\operatorname{Sm} A$ phase possess a wide range of soft mode in both the $\mathrm{Sm} C^{*}$ and $\mathrm{Sm} A$ phases. On reducing temperature limit of the $\mathrm{Sm} A$ phase, the regime of soft mode also shrinks towards the transition point of the $\mathrm{Sm}$ $C^{*}$ to $\mathrm{Sm} A$ phase. The influence of the $\mathrm{Sm} A$ phase temperature range on the soft mode can be explained theoretically by considering the coupling between the order parameters of two phases and the applied electric field using Landau's free-energy density expression as follows.

The free-energy density $g(z)$ is given by a Landau expansion in order parameters $\theta$ (tilt angle), $P$ (polarization), and $\varphi\left(\mathrm{Sm} A\right.$ order parameter). ${ }^{3,7}$ Assuming the coupling between the primary order parameters of the $\mathrm{Sm} C^{*}$ and $\mathrm{Sm}$ $A$ phases under the application of strong bias, the $g(z)$ can be written as

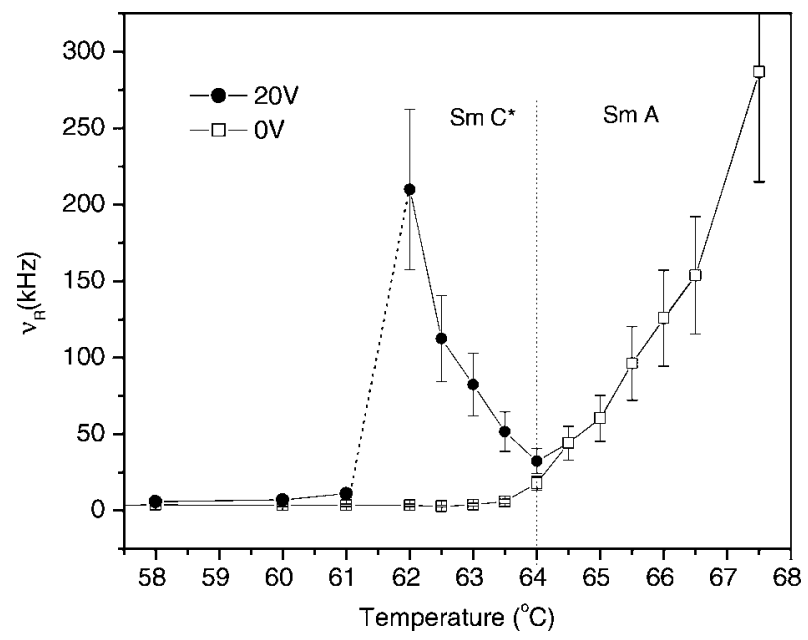

FIG. 7. Temperature dependence of relaxation frequency $\left(v_{R}\right)$ at 0 and $10 \mathrm{~V}$ biases in CS 1026. 


$$
\begin{aligned}
g(z)= & \alpha \varphi^{2}+\beta \varphi^{4}+a t \theta^{2} / 2+b \theta^{4} / 4+c \theta^{6} / 6-\Lambda \theta^{2} q \\
& +K \theta^{2} q^{2} / 3-d \theta^{4} q+P^{2} /(2 \varepsilon)+\eta P^{4} / 4-\mu P \theta q \\
& -C P \theta-\Omega P^{2} \theta^{2} / 2-E P-X \varphi^{2} \theta^{2},
\end{aligned}
$$

where the first five terms represent the free energy of the nonchiral $\mathrm{Sm} C$ phase with coefficients in Landau expansion of free-energy density. $\alpha$ and $\beta$ are the free-energy coefficients in the $\mathrm{Sm} A$ phase, $a$ is the temperature independent coefficient, $b$ and $c$ terms energy constants while $c$ term has been added to account for the specific heat temperature dependence of system. $t=\left(T-T_{c}\right) / T_{c}$ where $T$ is the temperature and $T_{c}$ is the transition temperature. $\Lambda$ is the coefficient of Lifshitz term responsible for modulation. $q$ represents the wave vector of energy density. $\mu$ and $C$ are the coefficient of flexo- and piezoelectric bilinear coupling terms. $\Omega$ is the coefficient of biquadratic coupling term. $\eta$ is added term to stabilize the system. The dielectric constant is $\varepsilon . K$ is the elastic modulus. $d$ is monotonic increase in pitch at low temperature. $E$ is the applied electric field. $X$ is the coupling coefficient used to describe the coupling between the order parameters of $\mathrm{Sm} C^{*}$ and $\mathrm{Sm} A .^{25}$ The other terms are included for the $\mathrm{Sm} C^{*}$ phase.

Minimizing Eq. (2) with respect to $\varphi$ and rewriting $g(z)$ in terms of $\theta$ and $P$,

$$
\begin{aligned}
g(z)= & -\alpha^{2} /(4 \beta)+[a t / 2+X \alpha /(2 \beta)] \theta^{2} \\
& +\left[b / 4-X^{2} /(4 \beta)\right] \theta^{4}+c \theta^{6} / 6-\Lambda \theta^{2} q+K \theta^{2} q^{2} / 3 \\
& -d \theta^{4} q+P^{2} /(2 \varepsilon)+P^{2} \eta / 4-\mu P \theta q-C P \theta \\
& -(1 / 2) \Omega P^{2} \theta^{2}-E P .
\end{aligned}
$$

Both order parameters can exist simultaneously in the Sm $C^{*}$ phase near $T_{c}$. Since the amplitude of Goldstone mode is very high, it is difficult to observe soft mode in the deep Sm $C^{*}$ phase even by suppressing Goldstone mode at high bias. However, this can be observed near $T_{c}$ in the $\mathrm{Sm}$ $C^{*}$ phase by suppressing Goldstone mode at high bias by clamping the dipole moments in one of the two possible states as the coupling between tilt and polarization becomes weak, which allows tilt fluctuations to occur.

On reducing the $\operatorname{Sm} A$ phase width, tilt-angle fluctuation would be induced more easily because the $\mathrm{Sm} A$ order parameter will not saturate at $T_{c}{ }^{7}$ Since tilt-angle fluctuations become easier, therefore it shows strong temperature dependence. The molecular tilt fluctuation becomes highly temperature dependent and so the soft mode frequency also becomes highly temperature dependent. Therefore, on increasing the width of the $\mathrm{Sm} A$ phase temperature range, the fluctuations condense and so the probability of relaxation existence in a broader temperature range in the vicinity of $T_{c}$ in both $\mathrm{Sm} C^{*}$ phase and $\mathrm{Sm} A$ phase becomes higher.

Relaxation frequency of soft mode in the $\mathrm{Sm} A$ phase using Eq. (3) can be written as ${ }^{3}$

$$
f_{S A} \approx \frac{\left\{\left[a\left(T-T_{c}\right)+X \alpha / \beta\right]+\left(K-\varepsilon \mu^{2}\right) q_{0}^{2}\right\}}{2 \pi} .
$$

On the basis of molecular field approximation, ${ }^{26}$ soft mode relaxation frequency in the $\mathrm{Sm} C^{*}$ phase can be written as

$$
f_{S C} \approx\left[a\left(T-T_{C}\right)+X \alpha / \beta\right]+\left[b-X^{2} / \beta\right] .
$$

In all four materials, it appears that the $P_{s}$ value of material do not influence the $\mathrm{Sm} C^{*}$-Sm $A$ phase transition like the width of the $\mathrm{Sm} A$ phase temperature range. As can be seen from the behavior of tilt and the DSC scans, the order of transition follows the behavior of the width of the $\mathrm{Sm} A$ phase, i.e., the reduction of width of the $\operatorname{Sm} A$ phase tends to favor first order transition while it is the second order transition that occurs for material for a larger width. From the free-energy expression [Eq. (3)], the behavior of order of transition is determined by $b^{\prime}=b / 4-X^{2} /(4 \beta)$. The transition becomes first order when $b^{\prime}$ becomes negative, and second order when it is positive. With the narrowing of temperature width in the $\mathrm{Sm} A$ phase, the coupling constant $X$ will be stronger due to the easiness of tilt fluctuations because the $\mathrm{Sm} A$ order parameter will not attain saturation at the Sm $C^{*}$-Sm $A$ phase transition. ${ }^{7,25}$ As can be seen from DSC scans, the degree of ordering from the $\mathrm{Sm} C^{*}$ to $\mathrm{Sm} A$ phase in Felix 17/100 drops much faster and to a larger extent as compared to other three materials; while it decreases with increase in width of the $\mathrm{Sm} A$ phase range exhibiting $N$ phase in sequence. Also, $a^{\prime}=a / 2+X \alpha /(4 \beta)$ describes the behavior of soft mode in the $\mathrm{Sm} A$ and $\mathrm{Sm} C^{*}$ phases according to Eqs. (4) and (5), respectively. The strong temperature dependence of tilt fluctuations can be seen in Eqs. (4) and (5) as the contribution of $X$ increases on narrowing the width of the $\operatorname{Sm} A$ phase temperature range. Felix 17/100, a FLC material with no observable soft mode contribution, raises a fundamental question over the existence of soft mode in the vicinity of the $\operatorname{Sm} C^{*}$-Sm $A$ phase as suggested by Levstik et $a l^{2}$

\section{CONCLUSION}

We have presented the experimental results of the $\operatorname{Sm} A$ phase temperature dependence of soft mode near the $\mathrm{Sm}$ $C^{*}$-Sm $A$ phase transition in four FLC materials of different $\mathrm{Sm} A$ phase widths. It has been observed that the probability of soft mode is less in a material having a very small temperature range of the $\mathrm{Sm} A$ phase. On the other hand, it is more for materials with a wide $\mathrm{Sm} A$ phase width. We interpret the observation as an easiness of tilt fluctuations with a narrowing $\mathrm{Sm} A$ phase width, which makes the coupling constant of order parameters of the $\operatorname{Sm~} C^{*}$-Sm $A$ phase stronger. This also affects the Landau free-energy density expression constants of second and fourth order terms, which describe the relaxation frequency in the $\operatorname{Sm} C^{*}$ phase and order of the $\operatorname{Sm} C^{*}$-Sm $A$ phase transition respectively.

\section{ACKNOWLEDGMENTS}

The authors are sincerely thankful to Dr. Vikram Kumar, Director, National Physical Laboratory for continuous encouragement and interest in this work. The authors (S.K. and J.P.) are thankful to CSIR, New Delhi for financial assistance. The authors are thankful to Gautam Singh and Indrani Coondoo for useful discussions. They are thankful to the Department of Science and Technology (DST), New Delhi 
for supporting this work under Project No. SR/S2/CMP-03/ 2005.

${ }^{1}$ R. B. Meyer, L. Liebert, L. Strzelecki, and P. Keller, J. Phys. (Paris), Lett. 36, L69 (1975).

${ }^{2}$ A. Levstik, T. Carlsson, C. Filipic, I. Levistik, and B. Zeks, Phys. Rev. A 35, 3527 (1987).

${ }^{3}$ T. Carlsson, B. Zeks, C. Filipic, and A. Levistik, Phys. Rev. A 42, 877 (1990).

${ }^{4}$ H. Y. Liu, C. C. Huang, Ch. Bahr, and G. Heppke, Phys. Rev. Lett. 61, 345 (1988).

${ }^{5}$ J. Boerio-Goates, C. W. Garland, and R. Shashidhar, Phys. Rev. A 41, 3192 (1990).

${ }^{6}$ C. W. Garland and M. E. Huster, Phys. Rev. A 35, 2365 (1987).

${ }^{7}$ C. C. Huang and S. C. Lien, Phys. Rev. Lett. 47, 1917 (1981).

${ }^{8}$ C. C. Huang and J. M. Viner, Phys. Rev. A 25, 3385 (1982).

${ }^{9}$ H. Y. Liu, C. C. Huang, T. Min, M. D. Wand, D. M. Walba, N. A. Clark, Ch. Bahr, and G. Heppke, Phys. Rev. A 40, 6759 (1989).

${ }^{10}$ C. C. Huang and S. C. Lien, Phys. Rev. A 31, 2621 (1985).

${ }^{11}$ S. K. Prasad, V. N. Raja, D. S. Shankar Rao, G. G. Nair, and M. E. Neubert, Phys. Rev. A 42, 2479 (1990).
${ }^{12}$ B. R. Ratna, R. Shashidhar, G. G. Nair, S. K. Prasad, Ch. Bahr, and G. Heppke, Phys. Rev. A 37, 1824 (1988).

${ }^{13}$ A. M. Biradar, S. Wrobel, and W. Haase, Phys. Rev. A 39, 2693 (1989).

${ }^{14}$ R. Shashidhar, B. R. Ratna, G. G. Nair, S. K. Prasad, Ch. Bahr, and G. Heppke, Phys. Rev. Lett. 61, 547 (1988).

${ }^{15}$ Ch. Bahr and G. Heppke, Mol. Cryst. Liq. Cryst. 150b, 313 (1987).

${ }^{16}$ R. B. Meyer, Mol. Cryst. Liq. Cryst. 40, 33 (1977).

${ }^{17}$ S. C. Lien, C. C. Huang, and J. W. Goodby, Phys. Rev. A 29, 1371 (1984).

${ }^{18}$ C. C. Huang and S. Dumrongrattana, Phys. Rev. A 34, 5020 (1986).

${ }^{19}$ S. Hiller, A. M. Biradar, S. Wrobel, and W. Haase, Phys. Rev. E 53, 641 (1996).

${ }^{20}$ J. Zubia, M. Castro, J. A. Puertolas, J. Etxebarria, M. A. Perez Jubindo, and M. R. de la Fuente, Phys. Rev. E 48, 1970 (1993).

${ }^{21}$ S. Kaur, A. K. Thakur, S. S. Bawa, and A. M. Biradar, Physica B 344, 133 (2004).

${ }^{22}$ A. K. Thakur, A. Choudhary, S. Kaur, S. S. Bawa, and A. M. Biradar, J. Appl. Phys. 100, 034104 (2006).

${ }^{23}$ T. Carlsson and I. Dahl, Mol. Cryst. Liq. Cryst. 95, 373 (1983).

${ }^{24}$ A. Mikulko, R. Douali, Ch. Legrand, M. Marzec, S. Wrobel, and W. Haase, Ferroelectrics 343, 133 (2006).

${ }^{25}$ L. Benguigui, J. Phys. (Paris), Colloq. 40, C3-419 (1979).

${ }^{26}$ R. Blinc and B. Zeks, Phys. Rev. A 18, 740 (1978). 